\title{
Approaches for Visualizing the Presence of Absence in Cartography
}

\author{
Anthony C. Robinson \\ GeoVISTA Center, Department of Geography, The Pennsylvania State University, - arobinson@psu.edu
}

Keywords: geovisual analytics, uncertainty, missing data, visualization

\begin{abstract}
:
Cartographers are continually challenged to react and respond to new sources and varieties of (big) spatial data. A contemporary challenge that has developed in the meantime is the need to attend to and make salient conspicuous absences in spatial data (Robinson, 2019). For example, traffic sensors that fail and return null values rather than valid data, or sudden drop in the number of visitors to a grocery store compared to a typical week may be in fact indicators of significant changes, but they may manifest in the form of null values or comparatively fewer observations to show on a map.
\end{abstract}

This scenario has been driven home by the enormous disruptions caused by the COVID-19 pandemic beginning in December 2019 and continuing through the present moment. Behavioral interventions necessary to curb the spread of a pathogen cause a wide range of conspicuous absences to emerge. Daily traffic gridlock in major cities disappeared, thousands of flights were cancelled, and pollution from factories suddenly stopped. More subtle changes across space and place include shorter trips to shopping centers, empty playgrounds at schools, and differences in the night time illumination in places where traffic decreased and businesses closed (Liu et al., 2020).
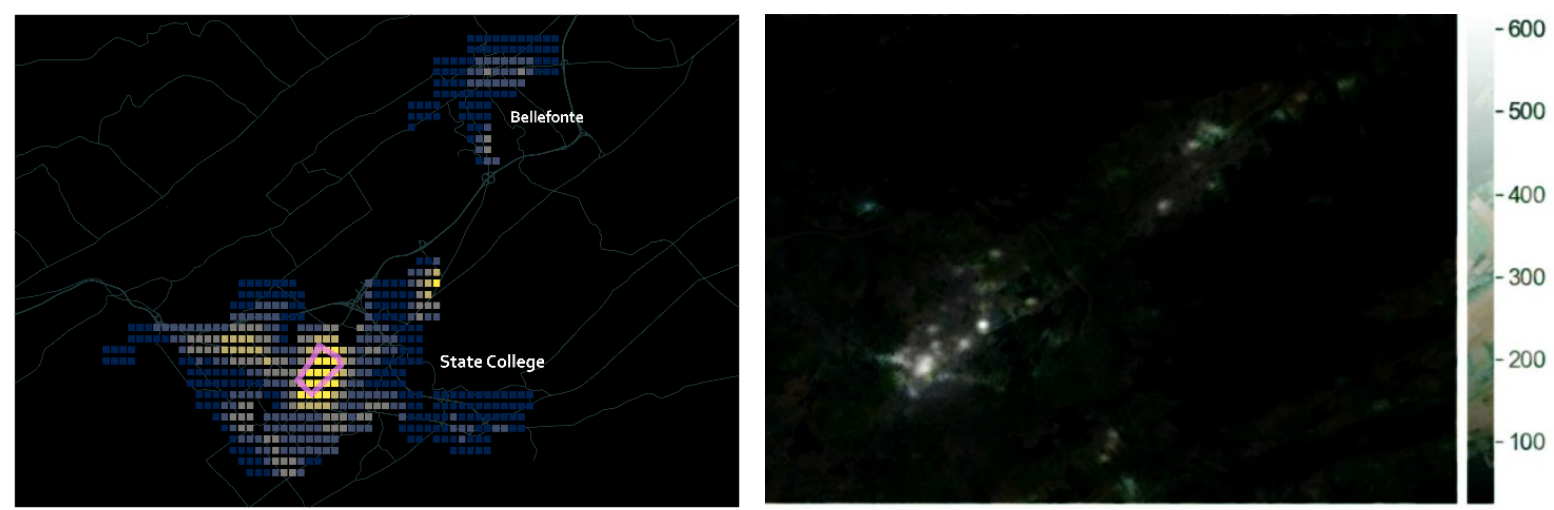

Figure 1. A wide range of visual approaches may be employed to signify absence in geovisualization. A gridded density symbolization is used at left, and a light blending with transparency is used at right. Both images depict changes in night-time luminance as detected by satellite imagery.

Those engaged in the development of geovisual analytics and cartographic solutions to problems in the contemporary world are likely to face similar challenges in the future where missing data and absence may be crucial signals to filter from and highlight just as outliers in a positive direction might be more commonly the focus of attention. A problem that emerges for the mapmaker in this context then regards how to visualize what is missing and how to communicate that information to readers.

On one hand, it is quite possible to signify absence exactly the way one signifies presence, and simply call it out as as such. Figure 1 shows this approach on the left side of the graphic, where changes in night-time lights in an area of Central Pennsylvania, USA are signified by the use of a light-dark color scheme (in this case, inverted with light representing high values and dark representing low values). It is also possible however to use absence more explicitly in the representational technique, as shown in Figure 1 on the right side of the graphic, where soft light blending is used to make luminance quite literally "light up" a layer of underlying satellite imagery. Darkness then represents where activity is missing, and luminance represents where activity is present.

A complicating factor for cartographers is the perceptual effect of visual asymmetry, which suggests that the presence of markers on an image is easier to perceive than for users to identify where such markers are absent (Wolfe, 2001). In 
mapping this effect is further complicated by the fact that we typically seek to overlay thematic data on top of base map information which may include reference data like roads, labels, and other basic symbology. It becomes even harder then to have negative space serve as a stand-in for absence/missingness in spatial data.

There are many avenues for future cartographic research that would expand what we know about visualizing the presence of absence. One of those focus areas could be the evaluation of visual approaches for signifying absence. Designing effective geovisualizations that highlight and integrate the presence of absence will require us to develop a better understanding regarding which techniques actually work to convey this presence to end users. Are some visual methods more semantically resonant with the concept of absence than others? Does semantic match have an impact on overall user performance or preferences? How can we make absence conspicuous as an outlier in its own right? What do we do when absence is diffuse across a map (e.g. all traffic is currently down $20 \%$ compared to last year) versus when it is concentrated and perhaps easier to visualize?

These are example research questions that lie ahead, and there are no doubt many more when one considers how to design geovisual interfaces, data storage schema, interactive techniques, and computational analysis methods that also require further attention to address this emerging area of concern. Previous work has focused a great deal of attention integrating uncertainty into geovisualization and reasoning with uncertain geographic information (Couclelis, 2003). Absence may in some ways be considered a type of uncertainty in spatial information. It would be wise to situate prior work in this area in light of what needs to happen to properly visualize absence going forward.

\section{References}

Couclelis, H. (2003). The Certainty of Uncertainty: GIS and the Limits of Geographic Knowledge. Transactions in GIS, $7(2), 165-175$.

Liu, Q., Sha, D., Liu, W., Houser, P., Zhang, L., Hou, R., Lan, H., Flynn, C., Lu, M., Hu, T., \& Yang, C. (2020). Spatiotemporal Patterns of COVID-19 Impact on Human Activities and Environment in Mainland China Using Nighttime Light and Air Quality Data. Remote Sensing, 12(10). https://doi.org/10.3390/rs12101576

Robinson, A. C. (2019). The Presence of Absence: Cartographic Representation for Missing Data. Annals of the American Association of Geographers, 109(1), 286-300. https://doi.org/10.1080/24694452.2018.1473754

Wolfe, J. M. (2001). Asymmetries in visual search: An introduction. Perception \& Psychophysics, 63(3), 381-389. https://doi.org/10.3758/bf03194406 